\title{
Suzaku observation of TeV blazar the 1ES 1218+304: clues on particle acceleration in an extreme $\mathrm{TeV}$ blazar
}

R. Sato ${ }^{a}$ J. Kataoka ${ }^{b}$ T. Takahashi ${ }^{a}$ G. M. Madejski ${ }^{c}$ S. Rügamer ${ }^{d}$ and S. J. Wagner ${ }^{e}$

E-mail: rsato@astro.isas.jaxa.jp

${ }^{a}$ Department of High Energy Astrophysics ISAS/JAXA, Sagamihara, Kanagawa 229-8510, Japan

${ }^{b}$ Department of Physics, Tokyo Institute of Technology, Meguro-ku, Tokyo 152-8551, Japan

${ }^{c}$ Stanford Linear Accelerator Center and Kavli Institute for Particle Astrophysics and

Cosmology, Stanford University, Stanford, CA 94305, USA

${ }^{d}$ Universität Würzburg, Am Hubland, D-97074 Würzburg, Germany

${ }^{e}$ Landessternwarte, Universität Heidelberg, Königstuhl, 69117 Heidelberg, Germany

\begin{abstract}
We observed the TeV blazar 1ES 1218+304 with the X-ray astronomy satellite Suzaku in May 2006. At the beginning of the two-day continuous observation, we detected a large flare in which the 5-10 keV flux changed by a factor of $\sim 2$ on a timescale of $5 \times 10^{4} \mathrm{~s}$. During the flare, the increase in the hard X-ray flux clearly lagged behind that observed in the soft X-rays, with the maximum lag of $2.3 \times 10^{4} \mathrm{~s}$ observed between the $0.3-1 \mathrm{keV}$ and $5-10 \mathrm{keV}$ bands. Furthermore we discovered that the temporal profile of the flare clearly changes with energy, being more symmetric at higher energies. From the spectral fitting of multi-wavelength data assuming a one-zone, homogeneous synchrotron self-Compton model, we obtain a magnetic field strength $B \sim 0.047$ $\mathrm{G}$, an emission region size $R=3.0 \times 10^{16} \mathrm{~cm}$ for an appropriate beaming with a Doppler factor of $\delta=20$. This value of $B$ is in good agreement with an independent estimate through the model fit to the observed time lag ascribing the energy-dependent variability to differential acceleration timescale of relativistic electrons provided that the gyro-factor $\xi$ is $10^{5}$.
\end{abstract}

Workshop on Blazar Variability across the Electromagnetic Spectrum

April 22-25 2008

Palaiseau, France 


\section{Observation and Data Reduction}

1ES 1218+304 was observed with Suzaku [14] during 2006 May 20-21 UT, yielding a net exposure time of 79.9 ks. Suzaku carries four sets of X-ray telescopes [17] each with a focal-plane X-ray CCD camera (XIS, X-ray Imaging Spectrometer; [13]) that is sensitive over the 0.3-12 keV band, together with a non-imaging Hard X-ray Detector (HXD; [12, 18]), which covers the 10-600 $\mathrm{keV}$ energy band with Si PIN photo-diodes and GSO scintillation detectors. 1ES 1218+304 was focused on the nominal center position of the HXD detector.

For the XIS, we analyzed the screened data, reduced via Suzaku software version 2.0. The screening was based on the following criteria: (1) only ASCA-grade 0,2,3,4,6 events were accumulated, while hot and flickering pixels were removed using the CLEANSIS script, (2) the time interval after the passage of South Atlantic Anomaly is greater than $500 \mathrm{~s}$, (3) the object is at least $5^{\circ}$ and $20^{\circ}$ above the rim of the Earth (ELV) during night and day, respectively. In addition, we also select the data with a cutoff rigidity (COR) larger than $6 \mathrm{GV}$. After this screening, the net exposure for good time intervals (GTIs) is $69.4 \mathrm{ks}$. The XIS events were extracted from a circular region with a radius of $4.2^{\prime}$ centered on the source peak, whereas the background was accumulated in an annulus with inner and outer radii of $5.4^{\prime}$ and $7.3^{\prime}$, respectively. We checked that the use of different source and background regions did not affect the analysis results. The response and auxiliary files are produced using the analysis tools XISRMFGEN and XISSIMARFGEN developed by the Suzaku team, which are included in the software package HEAsoft version 6.4.

The HXD/PIN data (version 2.0) were processed with basically the same screening criteria as those for the XIS, except that ELV $\geq 5^{\circ}$ through night and day and COR $\geq 8 \mathrm{GV}$. The HXD/PIN instrumental background spectra were provided by the HXD team for each observation ([12, 5]). Both the source and background spectra were made with identical GTIs and the exposure was corrected for detector deadtime of 6.0\%. We used the response file version AE_HXD_PINHXDNOM2_20070914.RSP.

\section{Analysis and Results}

Figure 1 shows the averaged light curves of the four XISs in the six X-ray energy bands. Although we could see variations of count rates at some level using HXD/PIN data, it was not significant within uncertainties of photon statistics. Thus in the following, we concentrate on the temporal variability of the XIS data only, below $10 \mathrm{keV}$. The temporal variation of the hardness ratio (HR) is also shown in the bottom panel of Figure 1. It indicates that the variability in the soft and hard X-ray bands are not well synchronized.

To quantify the different shape of the flare with energy dependent time-lags, we fitted the light curves with a function given by [15] after a slight modification of adding a constant offset $C_{0}$ to mimic the observed light curves:

$$
\begin{aligned}
I(t) & =C_{0}+C_{1} \times \exp \left[-\left(\left|t-t_{\text {peak }} / \sigma_{\mathrm{r}}\right|\right)^{k}\right] \quad\left(\text { for } t \leq t_{\text {peak }}\right), \\
& =C_{0}+C_{1} \times \exp \left[-\left(\left|t-t_{\text {peak }} / \sigma_{\mathrm{d}}\right|\right)^{k}\right] \quad\left(\text { for } t>t_{\text {peak }}\right),
\end{aligned}
$$

where $t_{\text {peak }}$ is the time of the flare's maximum intensity $C_{1}, k$ is a measure of pulse sharpness, $\sigma_{\mathrm{r}}$ and $\sigma_{\mathrm{d}}$ are the rise and decay time constants. If the light curve is symmetric in time, $\sigma_{\mathrm{r}}$ and $\sigma_{\mathrm{d}}$ 
are expected to be equal. All the light curves were binned at $2880 \mathrm{~s}$ (a half of the orbital period of Suzaku) for fitting. The results of the fittings are given in Table 1. In summary, the observed flare shows the following characteristics: (1) The flare shape is asymmetric in time $\left(\sigma_{\mathrm{r}} / \sigma_{\mathrm{d}}<1\right)$ especially in the lower energy band (but note $\sigma_{\mathrm{r}} / \sigma_{\mathrm{d}} \simeq 1$ for 5-10 keV light curve). (2) The flare amplitude defined as $\left(C_{1}+C_{0}\right) / C_{0}$ becomes larger as the photon energy increases (the 5-10 keV flux changed by a factor of $\sim 2$ ). (3) The rise-time of the flare is almost constant $\sim 5 \times 10^{4}$ s below $2 \mathrm{keV}$, while it becomes gradually longer at higher energy bands.

Next, we try to evaluate lags of temporal variations in various energy bands. Taking into account a wide variety of the flare shape measured at different energies, we estimated lags by just comparing the peak-time of the flare rather than using other temporal techniques, such as the discrete correlation function (DCF; [3] $)$ or the modified mean deviation method (MMD; [7]). ${ }^{1}$ We compared the peak-time in five lower energy bands to that determined in the 5-10 keV band. Apparently, the hard X-ray $(5-10 \mathrm{keV})$ peak lagged behind that in the soft X-ray $(0.3-1 \mathrm{keV})$ by $(2.3 \pm 0.7) \times 10^{4} \mathrm{~s}$. Importantly, this is much larger than the orbital period of Suzaku and less affected by artifacts proposed in [4].

The time averaged four XISs and HXD/PIN background subtracted spectra were fitted using XSPEC ver.11.3.2, including data within the energy band $0.6-50 \mathrm{keV}$. The background of HXD/PIN includes both the instrumental (non X-ray) background and the contribution from the cosmic X-ray background (CXB; [6]). Here the form of the CXB was taken as $9.0 \times 10^{-9}(\mathrm{E} / 3$ $\mathrm{keV})^{-0.29} \exp (-\mathrm{E} / 40 \mathrm{keV}) \operatorname{erg~} \mathrm{cm}^{-2} \mathrm{~s}^{-1} \mathrm{sr}^{-1} \mathrm{keV}^{-1}$ and the observed spectrum was simulated assuming the PIN detector response to isotropic diffuse emission. We first fitted with a single power-law model with Galactic absorption $N_{\mathrm{H}}=1.78 \times 10^{20} \mathrm{~cm}^{-2}$ [2]. We obtained the best fit photon index $\Gamma=2.14 \pm 0.01$, but this model did not represent the spectrum well yielding a reduced $\chi^{2}$ of 1.23 for 1967 dof. We then tried to fit with a broken power-law with Galactic absorption. The photon indices are $\Gamma_{1}=2.04 \pm 0.01$ and $\Gamma_{2}=2.17 \pm 0.01$ below and above the break energy $E_{\text {brk }}=1.42 \pm 0.05 \mathrm{keV}$, respectively. The flux over $2-10 \mathrm{keV}$ is $\sim 2.0 \times 10^{-11} \mathrm{erg} \mathrm{cm}^{-2} \mathrm{~s}^{-1}$. This model gives a better fit with a reduced $\chi^{2}$ of 1.14 for 1959 dof compared to the single power-law model, but $\chi^{2}$ is still not acceptable. Considering the spectral variability, we analyzed the spectrum every $5760 \mathrm{~s}$. The power-law indices vary from $2.05 \pm 0.01$ to $2.22 \pm 0.01$ during the flare, and each segment can be fitted well with a single power-law model or broken power-law model with $\chi^{2} /$ dof ranging from 0.94 to 1.09 .

Figure 2 shows the spectral energy distribution (SED) of 1ES 1218+304 with currently available datasets. As expected from the curved X-ray spectrum with $\Gamma_{1,2}$ around 2 and $E_{\text {cut }} \lesssim 10 \mathrm{keV}$, the synchrotron emission peaks just around the Suzaku bandpass. In order to specify the SED of 1ES 1218+304, we applied a one-zone homogeneous SSC model developed in [9]. Noting that the characteristic variability time scale of the flare is $t_{\mathrm{var}} \simeq 5 \times 10^{4} \mathrm{~s}$ (Figure 1), which is most probably determined by the light travel time across the source emitting region (see discussion in $\S 4$ ), we obtain $R=c t_{\mathrm{var}} \delta=3.0 \times 10^{16} \mathrm{~cm}$ for a moderate beaming factor of $\delta=20$ (e.g., [9, 10] for selfconsistent determination of physical parameters in TeV blazars). The resulting parameters are listed in Figure 2 caption. We note that the energy densities of electrons and fields are $u_{e}=8.3 \times 10^{-3}$

\footnotetext{
${ }^{1}$ Since the DCF quantifies the degree of similarity or correlation between two time series as a function of the timelag, it is not suitable to evaluate "energy-dependent" profiles, as observed in 1ES 1218+304.
} 


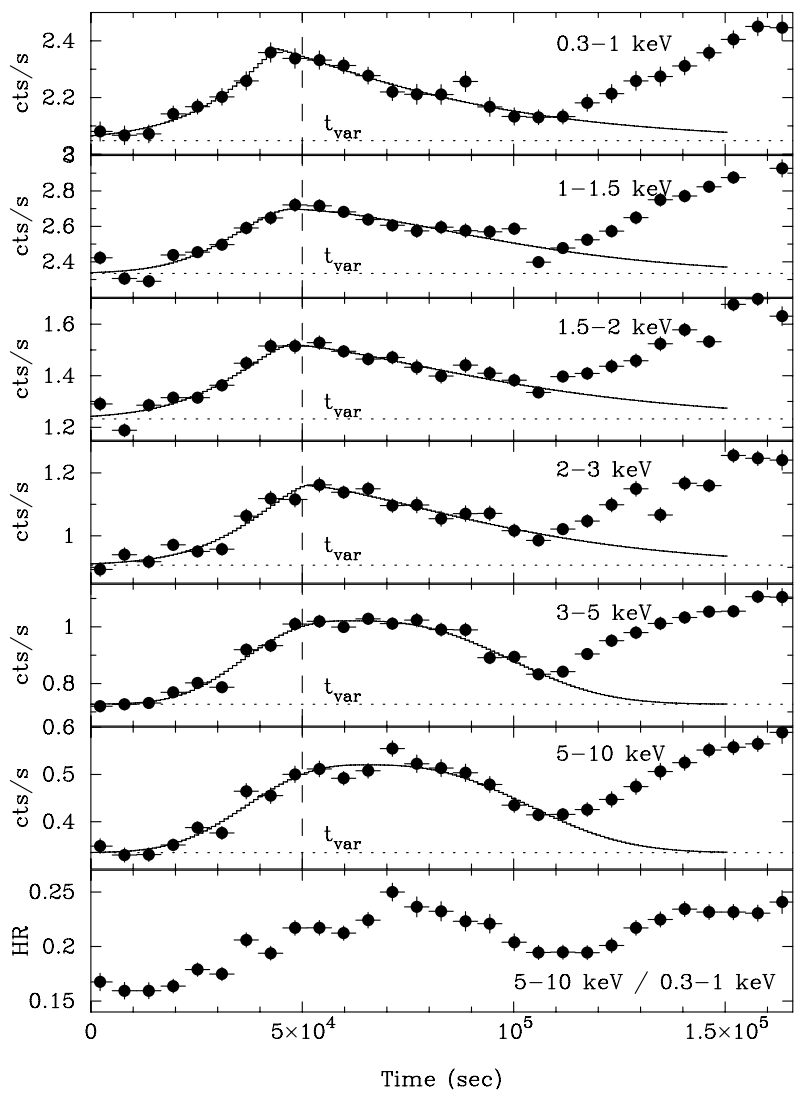

Figure 1: Suzaku XIS Light curves in the six energy bands: 0.3-1, 1-1.5, 1.5-2, 2-3, 3-5 and 5-10 keV (from the upper panel), respectively. The bottom panel shows the HR of count rates, defined as $(5-10 \mathrm{keV}) /(0.3-1$ $\mathrm{keV}$ ). The dotted line is the constant offset $C_{0}$. The dashed line shows $t_{\mathrm{var}} \simeq 5 \times 10^{4} \mathrm{~s}$.

Table 1: Flare shape of 1ES 1218+304 in various X-ray energies.

\begin{tabular}{lcccc}
\hline \hline $\mathrm{E}(\mathrm{keV})$ & $t_{\text {peak }}\left(10^{4} \mathrm{~s}\right)$ & $\sigma_{\mathrm{r}} / \sigma_{\mathrm{d}}$ & $k$ & $\left(C_{1}+C_{0}\right) / C_{0}$ \\
\hline $0.3-1$ & $4.4 \pm 0.1$ & $0.34 \pm 0.14$ & $1.2 \pm 0.4$ & $1.2 \pm 0.1$ \\
$1-1.5$ & $4.7 \pm 0.3$ & $0.31 \pm 0.08$ & $1.7 \pm 0.5$ & $1.2 \pm 0.1$ \\
$1.5-2$ & $4.7 \pm 0.3$ & $0.31 \pm 0.11$ & $1.4 \pm 0.5$ & $1.2 \pm 0.1$ \\
$2-3$ & $5.1 \pm 0.3$ & $0.33 \pm 0.10$ & $1.4 \pm 0.4$ & $1.3 \pm 0.1$ \\
$3-5$ & $6.1 \pm 0.4$ & $0.67 \pm 0.12$ & $2.7 \pm 0.4$ & $1.4 \pm 0.1$ \\
$5-10$ & $6.7 \pm 0.7$ & $0.84 \pm 0.17$ & $2.8 \pm 0.6$ & $1.6 \pm 0.1$ \\
\hline
\end{tabular}




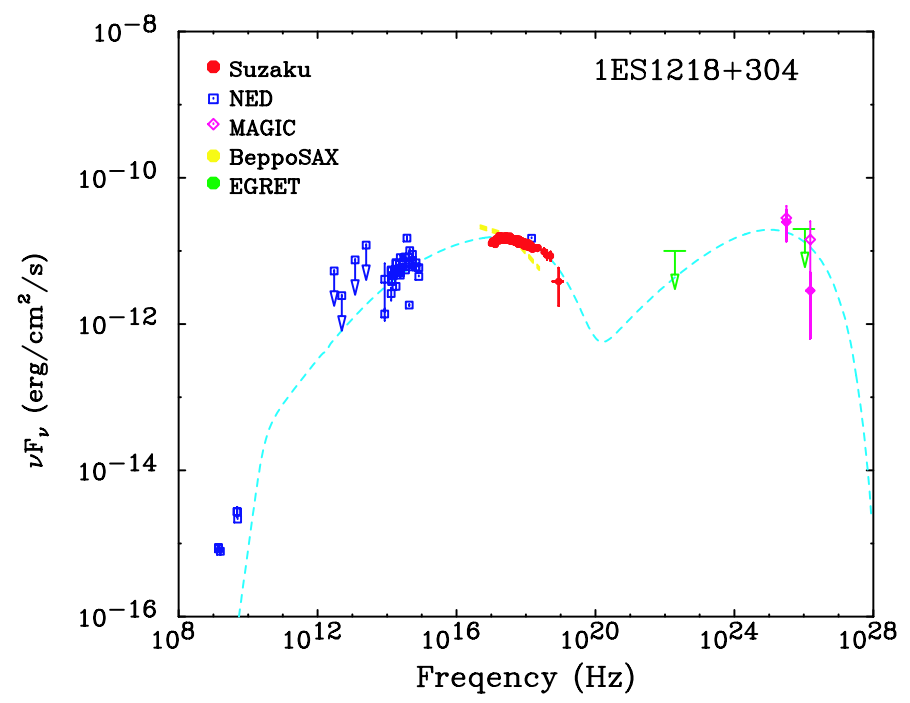

Figure 2: SED of 1ES 1218+304. The dashed line shows a one-zone SSC model assuming the parameters: $B=0.047 \mathrm{G}, \delta=20, s=1.7, \gamma_{\min }=1, \gamma_{\text {brk }}=8 \times 10^{3}$ and $\gamma_{\max }=8 \times 10^{5}$, where $s$ is the electron spectral index. See [16] for more detail.

erg $\mathrm{cm}^{-3}$ and $u_{B}=8.8 \times 10^{-5} \mathrm{erg} \mathrm{cm}^{-3}$, respectively. Thus the jet in $1 \mathrm{ES} 1218+304$ is particle dominated, and the ratio $u_{e} / u_{B} \sim 100$ is well within the range of typical TeV blazars.

\section{Discussion}

In our observation we detected a large flare during which the hard X-ray variation lagged behind that in the soft X-rays, $\sim 2.3 \times 10^{4} \mathrm{~s}$. This is completely opposite to a well-known "softlag", as has been obtained from the past observations. In the theoretical context, however, hard-lag is actually expected especially in the X-ray variability of TeV blazars, but has never been observed so clearly before. It has been suggested that a hard-lag is observable only at energies closer to the maximum electron energy, $\gamma_{\max }[11]$, where the acceleration time is almost comparable to the cooling time scale of radiating electrons: $t_{\text {acc }}\left(\gamma_{\max }\right) \simeq t_{\text {cool }}\left(\gamma_{\max }\right)$.

It is convenient to express $t_{\mathrm{acc}}$ and $t_{\mathrm{cool}}$ in terms of the observed photon energy $E$ (in units of $\mathrm{keV})$. Noting that the typical synchrotron emission frequency, averaged over pitch angles, of an electron with energy $\gamma m c^{2}$ is given by $v \sim 3.7 \times 10^{6} B \gamma^{2} \mathrm{~Hz}$, we obtain;

$$
\begin{aligned}
t_{\mathrm{acc}}(E) & =9.65 \times 10^{-2}(1+z)^{3 / 2} \xi B^{-3 / 2} \delta^{-3 / 2} E^{1 / 2} \mathrm{~s}, \\
t_{\text {cool }}(E) & =3.04 \times 10^{+3}(1+z)^{1 / 2} B^{-3 / 2} \delta^{-1 / 2} E^{-1 / 2} \mathrm{~s},
\end{aligned}
$$

where $z$ is the redshift, $\xi$ is the "gyro-factor" which can be identified with the ratio of energy in an ordered magnetic field to that in a turbulent magnetic field ( $\xi=1$ for the Bohm limit; see, e.g., [8]). Note that for lower energy photons, $t_{\text {acc }}(E)$ is always shorter than $t_{\text {cool }}(E)$ because higher energy electrons need longer time to be accelerated $\left(t_{\text {acc }}(\gamma) \propto \gamma\right)$ but cool rapidly $\left(t_{\text {cool }}(\gamma) \propto \gamma^{-1}\right)$. This energy dependence of acceleration/cooling time-scales may qualitatively explain the observed characteristics of the X-ray light curves of 1ES 1218+304. It is thus interesting to consider a simple 

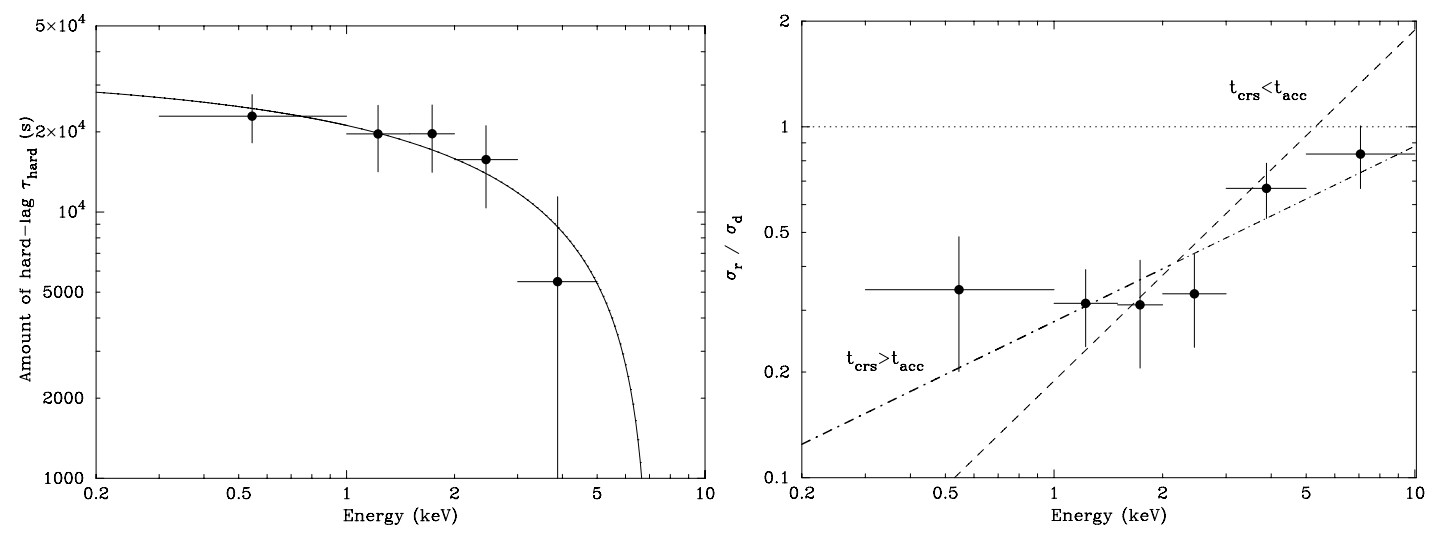

Figure 3: Left: Time lag of photons of various $\mathrm{X}$-ray energy bands vs $5-10 \mathrm{keV}$ band. The solid line corresponds to a fit with $\tau_{\text {hard }}=9.65 \times 10^{-2}(1+z)^{3 / 2} \xi B^{-3 / 2} \delta^{-3 / 2}\left(7.1^{1 / 2}-E_{\text {low }}^{1 / 2}\right)$, where $\delta$ is fixed to 20 . Right: Energy dependence of the pulse shape, defined as the ratio of $\sigma_{\mathrm{r}}$ and $\sigma_{\mathrm{d}}$. The dashed line shows the predicted value of $\sigma_{\mathrm{r}} / \sigma_{\mathrm{d}}$. The dash-dotted line shows $t_{\mathrm{crs}} / t_{\mathrm{cool}}$.

toy model in which the rise time of the flare is primarily controlled by the acceleration time of the electrons corresponding to observed photon energies, while the fall time of the flare is due to the synchrotron cooling time scale. In this model, the amount of hard-lag, $\tau_{\text {hard }}$, is simply due to the difference of $t_{\mathrm{acc}}$, and independent of the energy dependence of $t_{\mathrm{cool}}$ :

$$
\begin{aligned}
\tau_{\text {hard }} & =t_{\mathrm{acc}}\left(E_{\mathrm{hi}}\right)-t_{\mathrm{acc}}\left(E_{\mathrm{low}}\right) \\
& \sim 9.65 \times 10^{-2}(1+z)^{3 / 2} \xi B^{-3 / 2} \delta^{-3 / 2}\left(E_{\mathrm{hi}}^{1 / 2}-E_{\text {low }}^{1 / 2}\right) \mathrm{s},
\end{aligned}
$$

where $E_{\text {low }}$ and $E_{\text {hi }}$ are the lower and higher X-ray photon energies to which the time-lag is observed. Here we took $E_{\text {low } / \text { hi }}$ to be the logarithmic mean energy in the observation energy bandpass. The result of the model fit to the observed $\tau_{\text {hard }}$ is shown in Figure 3 (left).

Assuming $\delta=20$ from multiband spectral fitting (see $\S 2$ ), the best fit parameter of the magnetic field $B$ can be written as $\sim 0.049 \xi_{5} \mathrm{G}$, where $\xi_{5}$ is the gyro-factor in units of $10^{5}$. Thus, in order to have the $B$ field required in the acceleration region consistent with that derived from the SED fitting, we infer $\xi \sim 10^{5}$. Such high value of $\xi$ is in fact consistent with that inferred by [o] for other blazars. With these parameters, the maximum synchrotron radiation energy $E_{\max }$, corresponding to $\gamma_{\max }$, is expected to be $\sim 5.3 \mathrm{keV}$. Hence, the above toy model qualitatively well represents the observed spectral/temporal features of 1ES 1218+304, in particular: (1) the synchrotron component peaks around the Suzaku XIS energy band in the multiband spectrum and (2) the observed light curve is symmetric in shape when measured at the high energy band, while being asymmetric (i.e., fall time longer than rise time) at the lower energy band. Figure 3 (right) compares the energy dependence of observed and modeled flare shapes, defined as the ratio of rise and decay time-scales, $\sigma_{\mathrm{r}} / \sigma_{\mathrm{d}}$. The dashed line shows the model prediction from $\sigma_{\mathrm{r}} / \sigma_{\mathrm{d}} \simeq t_{\mathrm{acc}} / t_{\mathrm{cool}}$ $=\left(E / E_{\max }\right)^{1 / 2} /\left(E / E_{\max }\right)^{-1 / 2} \sim E / 5.3 \mathrm{keV}$. Although the general trend is well reproduced, Figure 3 indicates that the observed rise time may have a bit longer time scale than expected from the model. The most natural interpretation for this is the smoothing of rapid variability by the source 
light crossing time scale $t_{\text {crs }}$ (e.g., [1, 10]). Hence if the acceleration time scale is shorter than the source crossing time, we expect $t_{\text {crs }}$ to smooth out $t_{\text {acc }}$. The dash-dotted line in Figure 3 (right) shows the ratio of the time scales of $t_{\mathrm{crs}} / t_{\mathrm{cool}}$. we can see that $t_{\mathrm{crs}}$ is longer than $t_{\mathrm{acc}}$ below $\sim 2 \mathrm{keV}$, but comparable or shorter above $\sim 2 \mathrm{keV}$. As a result, for 1ES 1218+304 it seems reasonable that the rise time of the flare is primary determined by the acceleration time of the electrons, while the fall time of the flare is due to the synchrotron cooling time scale. Only the rise time of the flare in the lower energy bands are dominated by $t_{\text {crs }}$, but further studies using more data are necessary to confirm this model.

\section{References}

[1] Chiaberge, M., \& Ghisellini, G. 1999, MNRAS, 306, 551

[2] Costamante, L., et al. 2001, A\&A, 371, 512

[3] Edelson, R. A., \& Krolik, J. H. 1989, proceedings of the International Astronomical Union Symposium, 134, 96

[4] Edelson, R., Griffiths, G., Markowitz, A., Sembay, S., Turner, M. J. L., \& Warwick, R. 2001, ApJ, 554, 274

[5] Fukazawa, Y., et al. 2006, Proc.SPIE, 6266, 75

[6] Gruber, D. E., Matteson, J. L., Peterson, L. E., \& Jung, G. V. 1999, ApJ, 520, 124

[7] Hufnagel, B. R., \& Bregman, J. N. 1992, ApJ, 386, 473

[8] Inoue, S., \& Takahara, F. 1996, ApJ, 463, 555

[9] Kataoka, J., et al. 1999, ApJ, 514, 138

[10] Kataoka, J., et al. 2000, ApJ, 528, 243

[11] Kirk, J. G., Rieger, F. M, \& Mastichiadis, A. 1998, A\&A, 333, 452

[12] Kokubun, M. et al. 2007, PASJ, 59, S53

[13] Koyama, K. et al. 2007, PASJ, 59, S23

[14] Mitsuda, K. et al. 2007, PASJ, 59, S1

[15] Norris, J. P., et al. 1996, ApJ, 459, 393 2001, AIP Conf. Proc., 558, 463

[16] Sato, R., Kataoka, J., T. Takahashi., Madejski, G. M., Rügamer ${ }^{d}$, S., \& Wagner, S. J. ApJL in press (astro-ph/0804.2529)

[17] Serlemitsos, P. J., et al. 2007, PASJ, 59, S9

[18] Takahashi, T. et al. 2007, PASJ, 59, S35 